\title{
Análisis de los factores que influyen en la explosividad del polvo de carbón en las minas subterráneas
}

MINING ENGINEERING

\section{Analysis of the factors that affect in the explosibility of coal dust in underground mines}

\author{
Baquero K.*, Blandón A.*, Molina J.*§ \\ * Universidad Nacional de Colombia, Sede Medellín \\ §kybaquer@gmail.com,asblando@unal.edu.co,jmmolina@unal.edu.co
}

(Recibido: Diciembre 14 de 2011 - Aceptado: Noviembre 19 de 2012)

\begin{abstract}
Resumen
Con el fin de establecer los factores relacionados con las características del carbón que influyen en la explosividad del polvo de carbón en las minas subterráneas, se tomaron muestras de polvo de carbón de diferentes minas en Antioquia, Boyacá y Norte de Santander, y de las asociaciones de litotipos de varios de los mantos de una de las minas de Antioquia. A las muestras de polvo de carbón se les efectuaron los análisis de explosividad, y a las de los mantos, se les hicieron los análisis próximos, granulométricos, petrográficos, del punto de ignición, e índice de molienda hardgrove (IMH).

Los resultados indican que la gran mayoría del polvo de carbón presente en las minas es explosivo y por lo tanto requiere de inertización con caliza (principalmente) en porcentajes mayores al $60 \%$. Se determinó que la composición petrográfica juega un papel preponderante, ya que los plies enriquecidos en componentes liptiníticos, tienen mayor explosividad que los que son ricos en vitrinitas. Los carbones con propiedades plásticas, debido a que tienen hidrocarburos libres y a que normalmente producen polvos muy finos, presentan altos índices de explosividad.
\end{abstract}

Palabras Clave: Carbón, Explosividad de polvos, Inertización, Macérales.

\begin{abstract}
In order to establish which factors concern with coal dust explosibility in the underground mines samples of coal dust of different mines in Antioquia, Boyaca y Norte de Santander and of lithotypes associations of several coal seams of mine from Antioquia were collected. Samples were under explosibility test, proximate analysis, sizing, petrographic, ignition point, and index of grinding hardgrove (IGH) to the coal seams samples.

The results indicate that the majority of coal dust present in the mines is explosive and for this reason it requires of inertization in percentages greater than $60 \%$, with limestone (principally). It was determined that petrographic composition plays a roll preponderant roll in explosibility, i.e., the plies richest in liptinitic components have major explosibility than those rich in vitrinites. The coals with plastic proprieties, due they have free hydrocarbons and produce normally dust very thin, present high indexes of explosibility
\end{abstract}

Keywords: Coal, Dust explosibility, Inertization, Macerals. 


\section{Introducción}

A pesar de las investigaciones a lo amplio del mundo sobre seguridad en las minas de carbón, aún ocurren explosiones que involucran muertes y heridos, (Dobroski et al. 1996; McKinney et al. 2002; Light et al. 2007). Para citar algunos ejemplos se resalta la explosión por metano y principalmente polvo de carbón de la mina UBB en Virginia Occidental Estados Unidos en el 2010 con 29 fatalidades, y dos accidentes en las minas de Colombia en Amagá y Norte de Santander con 95 fatalidades en los años 2010 y 2011.

Estudios experimentales por la oficina de seguridad minera e investigación en salud, en Estados Unidos y otras similares en diferentes países han mostrado que el polvo de roca inerte actúa como un disipante del calor y mezclado en suficiente cantidad con el polvo de carbón previenen las explosiones de polvo de carbón, (Cybulski 1975; Michelis et al. 1987, 1996; Lebecki 1991; Reed et al. 1989).

Las regulaciones establecen que las vías de minas de carbón bituminoso en Estados Unidos deben mantener un contenido de incombustible de al menos $65 \%$ en la corriente de ventilación entrante y de al menos $80 \%$ en la ventilación de retorno. Las vías de ventilación de retorno requieren más material inerte porque hay mayor riesgo de acumulación de polvo de carbón más fino. Las regulaciones en Estados Unidos también requieren un $1 \%$ adicional de incombustible por peso, por cada $0.1 \%$ de metano en la corriente de ventilación entrante y $0.4 \%$ adicional de incombustible por cada 0.1 $\%$ de metano en el retorno (CFR 30, 2011).

El contenido total de incombustible (CTI) incluye las medidas de humedad en las muestras, las cenizas en el carbón y el polvo de roca.

El 65 de CTI requerido para la ventilación entrante fue adoptado con base en los resultados de dos estudios. Primero las muestras de polvo de carbón fueron colectadas y medidas para determinar el tamaño promedio de las partículas de polvo de carbón. Después se hicieron pruebas en minas experimentales para determinar la cantidad de polvo de roca requerido para que las partículas de carbón del tamaño colectado en la investigación se volvieran inertes, (Nagy 1981).

El término polvo tamaño mina fue adoptado en la mitad de los 1920 y se refiere al polvo de carbón que pasa a través de una malla 20 US estándar $(850 \mu \mathrm{m})$ y contiene $20 \%$ pasante malla $200(75 \mu \mathrm{m})$. Las muestras colectadas de los pisos de las minas tienen de 5 a $40 \%$ del material pasante malla $200(75 \mu \mathrm{m})$. Las mallas empleadas en este artículo son inoxidables y se refiere a la norma ASTM E11 (ASTM, 2009).

Para el $80 \%$ de las minas, los valores finales variaron de 15 a $25 \%$ a pasante malla 200 . Los autores reconocen que el polvo colectado de las paredes, y el techo fue más fino, con 40 a $75 \%$ de las partículas más finas que malla 200.

Las cantidades de polvo de roca que se requieren en la ventilación de retorno en minas de carbón en los Estados Unidos fueron incrementadas al 80 $\%$. Además recomiendan que el polvo de carbón se deba limpiar y no se permite que se acumule en frentes de trabajo o en equipos eléctricos. Cuando hay excesivo polvo, se debe aplicar agua más un agente humectante, para evitar el polvo, especialmente a distancias menores que 40 pies del frente de explotación para minimizar el peligro de explosión, (Cashdóllar, Kennet L. et. al., 2010).

Se requiere que todas las áreas subterráneas donde el contenido de incombustible es muy bajo, se espolvoreen el polvo de roca 10 pies del frente de explotación.

Donde se requiera aplicar el polvo de roca, este debe ser distribuido sobre el techo, el piso y los lados de todas las áreas subterráneas de una mina de carbón y se mantiene en tales cantidades que el contenido de incombustible de la combinación polvo de carbón, polvo de roca y otros polvos no serán menos que el $65 \%$, pero el contenido de incombustible en el curso del aire de retorno no sea menos que el $80 \%$.

Los requerimientos para que el polvo de carbón haga explosión son: un polvo combustible, esparcido en el aire, a una concentración por encima del límite inflamable, la presencia de una fuente de ignición suficientemente energética 
y algo de confinamiento. La explosión de un combustible en el aire consiste en la oxidación rápida del material combustible, lo cual llega a un rápido aumento de la temperatura y la presión. La violencia de una explosión está relacionada a la tasa de energía liberada por la reacción química, en relación con el grado de confinamiento y la pérdida de calor (Cashdóllar, Kennet L. 2000).

Las propiedades de combustión del polvo de carbón dependen de las características químicas, físicas, y especialmente de la distribución del tamaño de partícula.

En este artículo se analizan los principales factores que influyen en la explosión de polvo de carbón a fin de entender los fenómenos que ocurren y generar conocimiento que permita prevenirla.

Explosividad del polvo de carbón en Colombia

En Colombia han ocurrido grandes desastres y explosiones catastróficas debido al polvo de carbón, tanto en minas subterráneas que explotan carbón térmico en la región de Antioquia como en donde se explota carbón metalúrgico de las regiones de Norte de Santader y Boyacá, lo cual da un indicio de la magnitud del problema.

Dadas las condiciones de informalidad de la minería subterránea del carbón, sólo hasta ahora empieza a estudiarse el trabajo relacionado con la explosividad del carbón desde el punto de vista técnico. Por medio de proyectos de grado en la Facultad de Minas, se han adelantado algunos estudios tales como los de (Castro W y Martínez C, 1997, 1998, 1999, 2000 y Baquero M. K. 2010), este último trabajo titulado, "Análisis de los factores que influyen en la combustión espontánea del carbón en una mina en Amagá", el cual consistió en analizar los factores que influyen en la combustión espontánea en los mantos de carbón en la mina, aspecto considerado como una fuente potencial de explosiones.

\section{Metodología}

La parte experimental de este estudio consiste básicamente en analizar los factores relacionados con las características del carbón que influyen en la susceptibilidad a la explosión del polvo de carbón en minas subterráneas. Para el efecto se tomaron muestras de cada uno de los ply de un Manto y del polvo de carbón en una mina del Municipio de Amagá. Además se tomaron otras muestras de polvo de carbón en minas de Norte de Santander, Boyacá y Antioquia. Se realizaron análisis próximos y se efectuaron análisis de explosividad mediante un sensor infrarojo certificado por la Oficina Nacional de Seguridad Minera de Estados Unidos, que se explicará adelante con mayor detalle.

\subsection{Muestreo y preparación}

\section{Mantos de carbón.}

Los mantos de carbón presentan variaciones verticales en el contenido y tipo de materia orgánica, por lo tanto, se empleó la técnica de muestreo de canal por ply, (Thomas 2002), la cual consiste en obtener un volumen de carbón triturado que incluya el espesor total de cada ply, mediante un corte uniforme, continuo y perpendicular a la estratificación. Para que la muestra fuera representativa, el muestreo de mantos de carbón se realizó en dos frentes de explotación de la mina, garantizando que las condiciones naturales del material estuvieran lo más frescas posibles (Figura 1).

\section{Polvos de carbón}

La toma de este tipo de muestra se efectuó en diferentes minas de Norte de Santander, Boyacá y Antioquia, en las paredes, techos y pisos de la zona de explotación. Estas muestras fueron recolectadas haciendo uso de una brocha y un recogedor plástico, acumulándose una cantidad suficiente de polvo de carbón, el cual se conservó en un frasco limpio, o en una bolsa plástica, dependiendo de la cantidad recolectada. Posteriormente, se efectuó el análisis de explosividad.

Para disminuir los errores en los resultados, se hace la siguiente preparación a las muestras de polvo de carbón tomadas: Se tamiza la muestra a pasante malla 20 y se adiciona tamiz molecular para eliminar la mayor cantidad de humedad. 


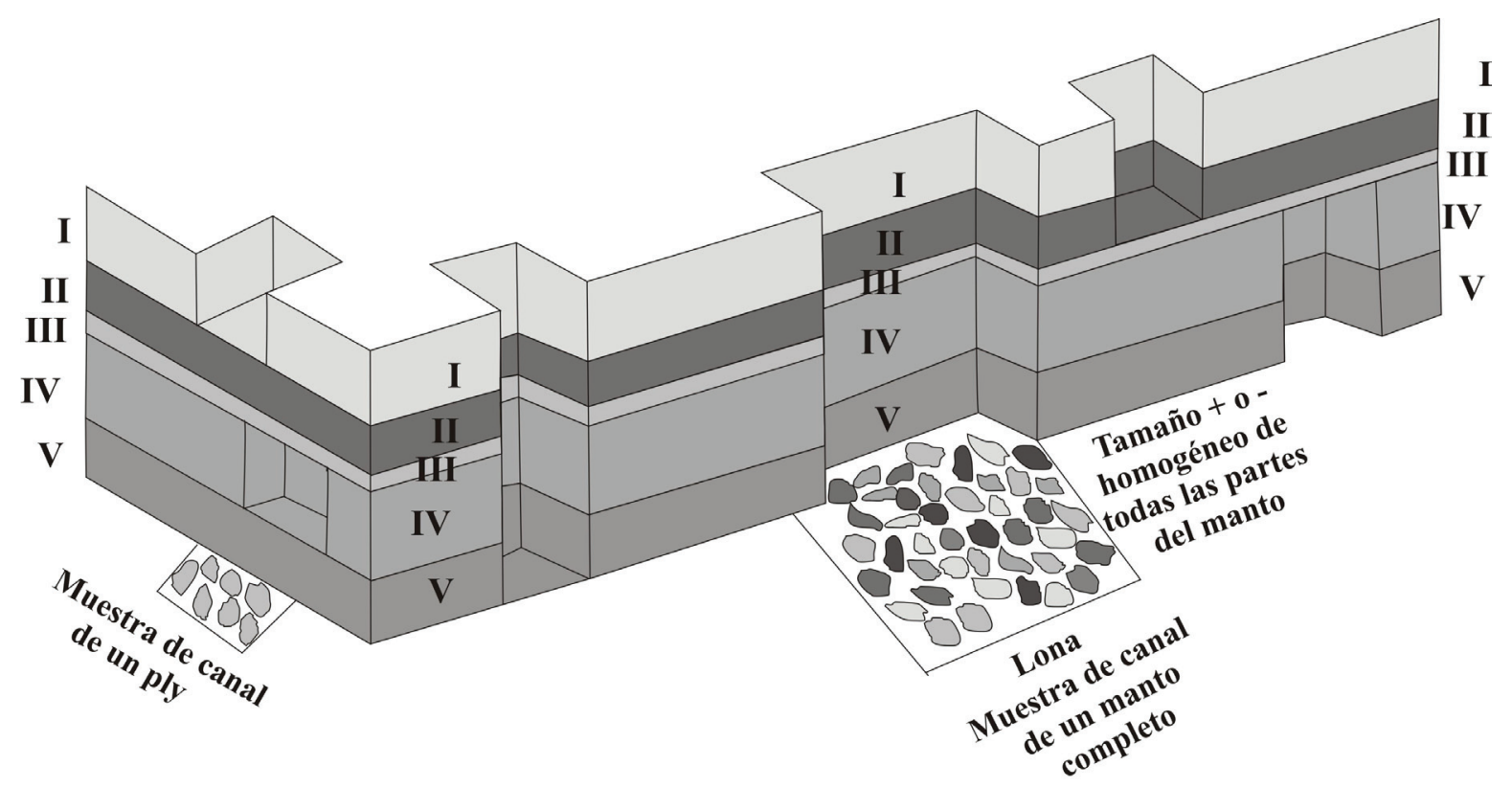

I, II, III, IV, y V = Ply (Variación vertical del manto)

Figura 1. Esquema de muestreo de canal en un manto de carbón. Por cada 20 a $30 \mathrm{~cm}$ de espesor del manto, se toman de 2 a $3 \mathrm{~kg}$ de muestra.

Con el fin de identificar el porcentaje de inertización hasta que dichos polvos no fuesen explosivos (Green); se realizaron varias mezclas con polvo de caliza, en diferentes proporciones. En la Tabla 1, se observan las mezclas por cantidades específicas de polvo de carbón y polvo de caliza y el indicador de explosividad para cada una de ellas.

Tabla 1. Mezclas por porcentaje y peso.

\begin{tabular}{ccccc}
\hline $\begin{array}{c}\text { \% Polvo } \\
\text { de Roca }\end{array}$ & $\begin{array}{c}\text { \% Polvo } \\
\text { de carbón }\end{array}$ & $\begin{array}{c}\text { Polvo de } \\
\text { Roca }(\mathrm{g})\end{array}$ & $\begin{array}{c}\text { Polvo de } \\
\text { Carbón }(\mathrm{g})\end{array}$ & $\begin{array}{c}\text { Total } \\
(\mathrm{g})\end{array}$ \\
\hline 85 & 15 & 42.5 & 7.5 & 50 \\
80 & 20 & 40 & 10 & 50 \\
75 & 25 & 37.5 & 12.5 & 50 \\
70 & 30 & 35 & 15 & 50 \\
\hline
\end{tabular}

\subsection{Ensayos de laboratorio}

Existen muchos métodos o ensayos por medio de los cuales se puede determinar la susceptibilidad del polvo de carbón a la explosión. Sin embargo, para esta investigación sólo se hicieron los análisis de un Manto de la una Mina en Amagá, nombrados en la Tabla 2, con las respectivas normas de ejecución a las muestras recolectadas.
Para el caso de las muestras de polvo de carbón de las minas de Norte de Santander y Boyacá, solo se efectuaron los ensayos de explosividad.

Análisis próximo de carbón

Según la norma ASTM, estos análisis se definen como el contenido de humedad residual, cenizas, materia volátil y carbono fijo. Sin embargo, también se realizan los análisis próximos completos, los cuales incluyen la determinación del poder calorífico y el azufre total.

Análisis granulométricos

Se hizo el análisis de distribución de tamaño de grano utilizando las mallas 70, 100, 140 y 200, dado que acorde con varios autores (Cybulski 1975; Michelis et al. 1987, 1996; Lebecki 1991; Reed et al. 1989) son los tamaños más representativos para observar el comportamiento a la explosividad de los carbones.

Análisis de índice de molienda hardgrove

Este análisis indica la facilidad con que el carbón se deja moler y por lo tanto, está relacionado al grado de dureza del carbón. 
Tabla 2. Análisis realizados a las muestras y sus respectivas normas de ejecución

\begin{tabular}{ccc}
\hline Análisis & Determinación & Normas \\
\hline Próximos completos & Humedad residual & ASTM D3173 \\
& Cenizas & ASTM D3174 \\
& Materia volátil & ASTM D3175 \\
& Carbono fijo & ASTM D3172 \\
Granulométricos & Azufre total & ASTM D4239 \\
Índice de Molienda Hardgrove & Poder calorífico & ASTM \\
Ignición & Tamaños $<70$ hasta $<200$ micras \\
Petrográficos & Grado de dureza del carbón \\
Explosividad & Temperatura de ignición del carbón y tiempo de quema \\
& Vitrinita, Liptinita , Interinita y Minerales
\end{tabular}

Pruebas de ignición del carbón

Con la realización de este análisis se pretende conocer la temperatura a la cual el carbón hace chispa por primera vez. Para ello se requiere preparar las muestras, y colocarlas en una pequeña porcelana dentro de una mufla a la cual se le aumenta gradualmente la temperatura, por cortos periodos de tiempo conocidos como tiempo de exposición. A medida que se avanzan en el proceso, es necesario monitorear las muestras. Con el fin de obtener resultados que ayuden a analizar los factores que influyen en la autoignición del carbón, las muestras usadas para esta prueba corresponden a las mallas $(70,100,140$ y 200$)$. Para todas las muestras, la temperatura inicial fue de $50{ }^{\circ} \mathrm{C}$ y se fue aumentando gradualmente $50^{\circ} \mathrm{C}$ hasta alcanzar la temperatura de ignición. En general se trabajó con un tiempo de exposición de 10 minutos en cada una de las temperaturas.

\section{Análisis petrográfico}

Este análisis se trata del estudio microscópico, para el cual se realizaron cuatro secciones pulidas con las muestras de carbón de los ply de un Manto de una Mina en Amagá, a partir de las cuales es posible identificar los diferentes macerales y minerales que constituyen el carbón y de esta manera poder clasificarlos. El método utilizado en este análisis, consiste en realizar un conteo de 500 puntos de los macerales y minerales presentes en la muestra, cuantificándolos por medio de porcentajes que indican ciertas condiciones de formación y rango del carbón, para esto se hizo necesario utilizar un microscopio petrográfico de luz reflejada normal y fluorescente y con objetivos especiales para aceite de inmersión y secos.

Análisis de explosividad de polvos de carbón

Para este ensayo se utilizó el medidor de explosividad de polvos de carbón (CDEM por su sigla en inglés Coal Dust Explosivility Meter), el cual fue desarrollado por National Institute for Occupational Safety and Health (NIOSH). Este es un instrumento manual que proporciona una medida directa del potencial de explosividad de polvos de carbón, independiente del tamaño de las partículas de carbón.

El funcionamiento de este instrumento consiste en un sensor óptico conectado a una pequeña caja electrónica con una pantalla digital, por medio del cual se realiza la medición de la radiación infrarroja reflejada por la superficie de una mezcla homogénea de dos sustancias con diferentes reflectancias ópticas, como el polvo de roca y el polvo de carbón. Además sirve como dispositivo complementario para mejorar la seguridad en las minas, ya que permite determinar la cantidad de polvo de caliza que es necesario adicionar al polvo de carbón para inertizarlo. Con este equipo se puede medir la explosividad de los polvos de carbón de manera cualitativa, es decir que los resultados arrojados por el dispositivo manual son tres colores, los cuales son indicadores del nivel de explosividad en términos de muy explosivo 
(red), moderadamente explosivo (yellow) y no explosivo (green), Harris et., al, 2008, Tabla 3.

Tabla 3. Indicadores del nivel de explosividad, (Harris et., al, 2008)

\begin{tabular}{cc}
\hline Indicador & Grado de explosividad \\
\hline Rojo & Altamente explosivo \\
Amarillo & Moderadamente explosivo \\
Verde & No explosivo \\
\hline
\end{tabular}

\section{Resultados y discusión}

A partir de los resultados obtenidos de los ensayos realizados a las muestras, se pretende conocer un poco más a cerca de losw factores que influyen en la susceptibilidad del polvo de carbón a la explosión, con el fin de priorizarlos y de este manera dar recomendaciones, tomando medidas de prevención que ayuden a mitigar los daños causados por las explosiones, al controlar estos factores que juegan un papel decisivo en el desarrollo del fenómeno. A continuación se presentan los resultados obtenidos de cada uno de los análisis realizados a las muestras.

En la tabla 4 se presentan los resultados de los análisis: próximos, granulométricos, de índice de molienda hardgrove, del grado de explosividad del polvo de carbón y los petrográficos de cuatro muestras correspondientes a la variación vertical del un Manto de una mina en Amagá.

En las figuras 2 a 7, se presentan los resultados de los análisis: próximos, granulométricos, de índice de molienda hardgrove, del grado de explosividad del polvo de carbón y los petrográficos de cuatro muestras correspondientes a la variación vertical del un Manto en una mina en Amagá. En las figuras 2 a 6 , se observan las relaciones que existen entre cada uno de los análisis antes mencionados y el grado de explosividad del polvo de carbón.

Tabla 4. Resultados de los análisis próximos de las muestras de los plies de un Manto de una Mina en Amagá

\begin{tabular}{cccccccccc}
\hline Muestra & HR & Cz & M.V & M.V (lac) & C.F & ST & P.C. & P.C (lac) & ÍMH \\
\hline M1 Ply 1 & 12.08 & 2.59 & 40.27 & 47.19 & 45.06 & 0.43 & 6176 & 7238 & 35.2 \\
M1 Ply 2 & 13.31 & 3.71 & 33.67 & 40.58 & 49.31 & 0.34 & 5751 & 6931 & 36.6 \\
M1 Ply 3 & 11.49 & 2.30 & 39.81 & 46.18 & 46.4 & 0.33 & 6197 & 7188 & 33.1 \\
M1 Ply 4 & 11.11 & 2.54 & 41.56 & 48.13 & 44.79 & 0.33 & 6402 & 7414 & 30.3 \\
\hline
\end{tabular}

$H R=$ Humedad residual; $C z=$ Cenizas; M.V. = Materia volátil; (lac) = Libre de agua y ceniza. $S T=$ Azufre total. P.C. $=$ Poder calorifico. $I M H=$ indice de molienda hardgrove

\subsection{Análisis próximos completos}

\subsubsection{Relación de los análisis próximos con el grado de explosividad}

Para muestras del mismo rango como las que se muestran en la tabla 4, hay una relación directa entre los análisis de materia volátil (lac), y poder calorífico (lac) con el grado de explosividad del polvillo de carbón. Así el ply 2 que presenta menor contenido de materia volátil y el menor poder calorífico, presenta el menor grado de explosividad, mientras que el ply 4 , el cual presenta el mayor contenido de materias volátiles y el mayor poder calorífico, tiene el mayor grado de explosividad. Véase figura 2.

\subsection{Análisis granulométrico}

A las muestras de polvos de carbón, también se les realizó un análisis granulométrico, con el fin de identificar con claridad los tamaños de material particulado predominante en la mina, además de conocer la influencia de este factor en la formación de nubes de polvo que generen explosiones, los resultados se muestran en las Figuras 3, 5, 6 y 7. Aquí se puede notar que la mayor cantidad de polvo de carbón en el de un manto de una mina en Amagá tiene tamaños 
que están entre pasante de malla $100 \mathrm{y}$ pasante de malla 200, indicando que la generación de polvos finos en la mina es alta. Además en la figura 6 , se identifica que es un factor que influye en la susceptibilidad del polvo de carbón a la explosión, dada la variación en la explosividad que presenta un mismo ply, por lo que los constituyentes del carbón se convierten en una fuente peligrosa para la ocurrencia de explosión.
En general los resultados revelan que entre más finos sean los polvos de carbón, estos son más explosivos, por lo que las partículas de polvo de carbón que pasan la malla 200 representan un grave peligro, lo cual hace necesario utilizar un alto porcentaje de material de inertización, para reducir el riesgo por explosiones de polvo en la mina.

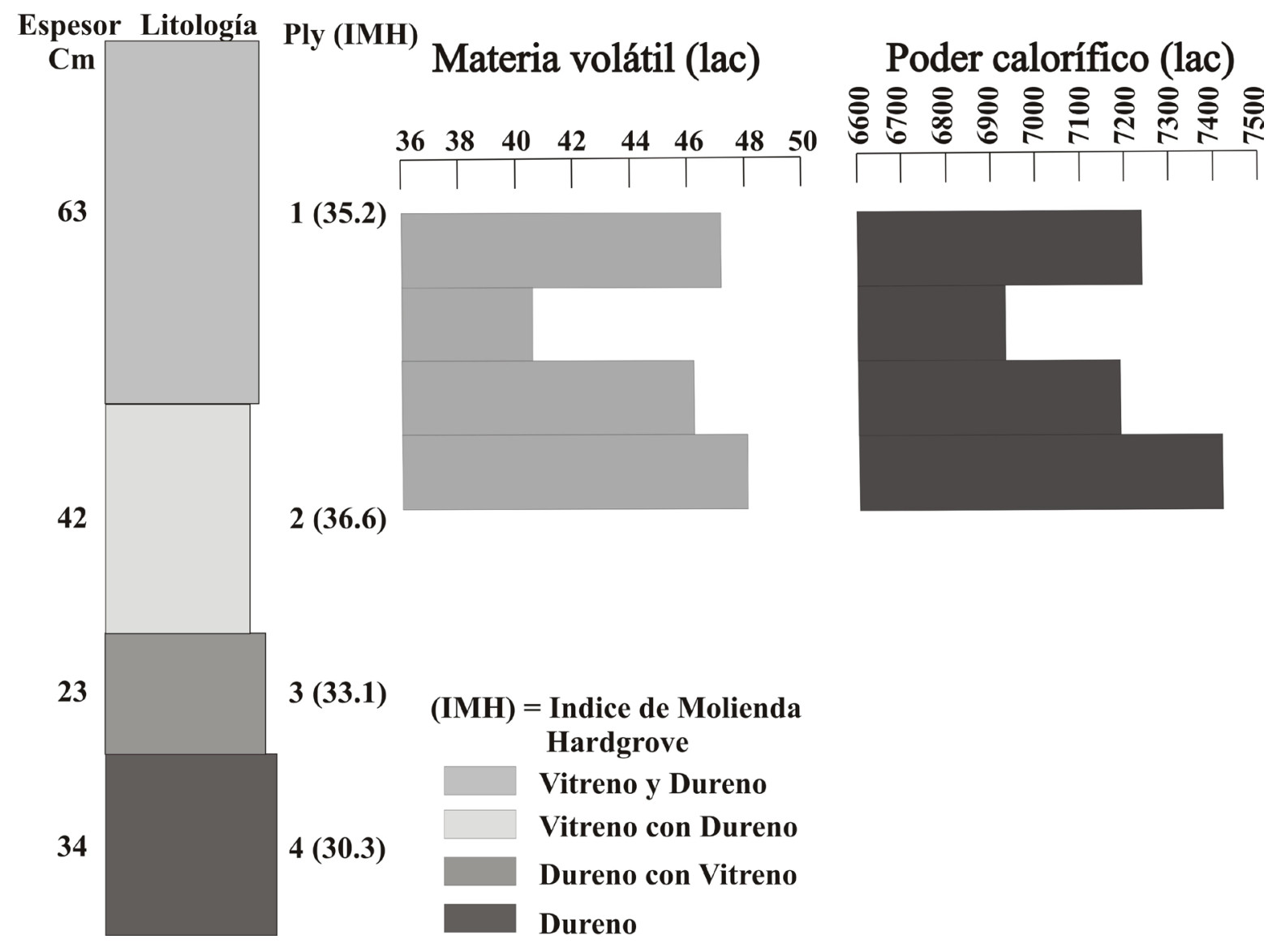

Figura 2. Relación entre el índice de molienda hardgrove y el contenido de materia volátil (lac) y el poder calorifico (lac) a lo largo del un manto de una Mina en Amagá

\subsection{Indice de molienda hardgrove (IMH)}

\subsubsection{Relación del tamaño de grano con el} índice de molienda y el grado de explosividad

En la figura 3 se puede observar que existe una relación directa entre el tamaño de grano y el índice de molienda, así el ply de carbón más blando (36.6) tiene mayor porcentaje de partículas finas $(<200)$, mientras que el ply más duro (30.3) tiene menor porcentaje de partículas
$(<200)$, pero el grado de explosividad en este caso no está directamente relacionado con el tamaño de las partículas, sino con la composición de dichas partículas, así el ply 4 más rico en dureno que significa que tiene mayor contenido de macerales liptiniticos, es el que presenta mayor grado de explosividad, mientras que el ply 2 que es el más rico en vitreno, es decir que tiene mayor cantidad de macerales vitriniticos, tiene menor grado de explosividad. 


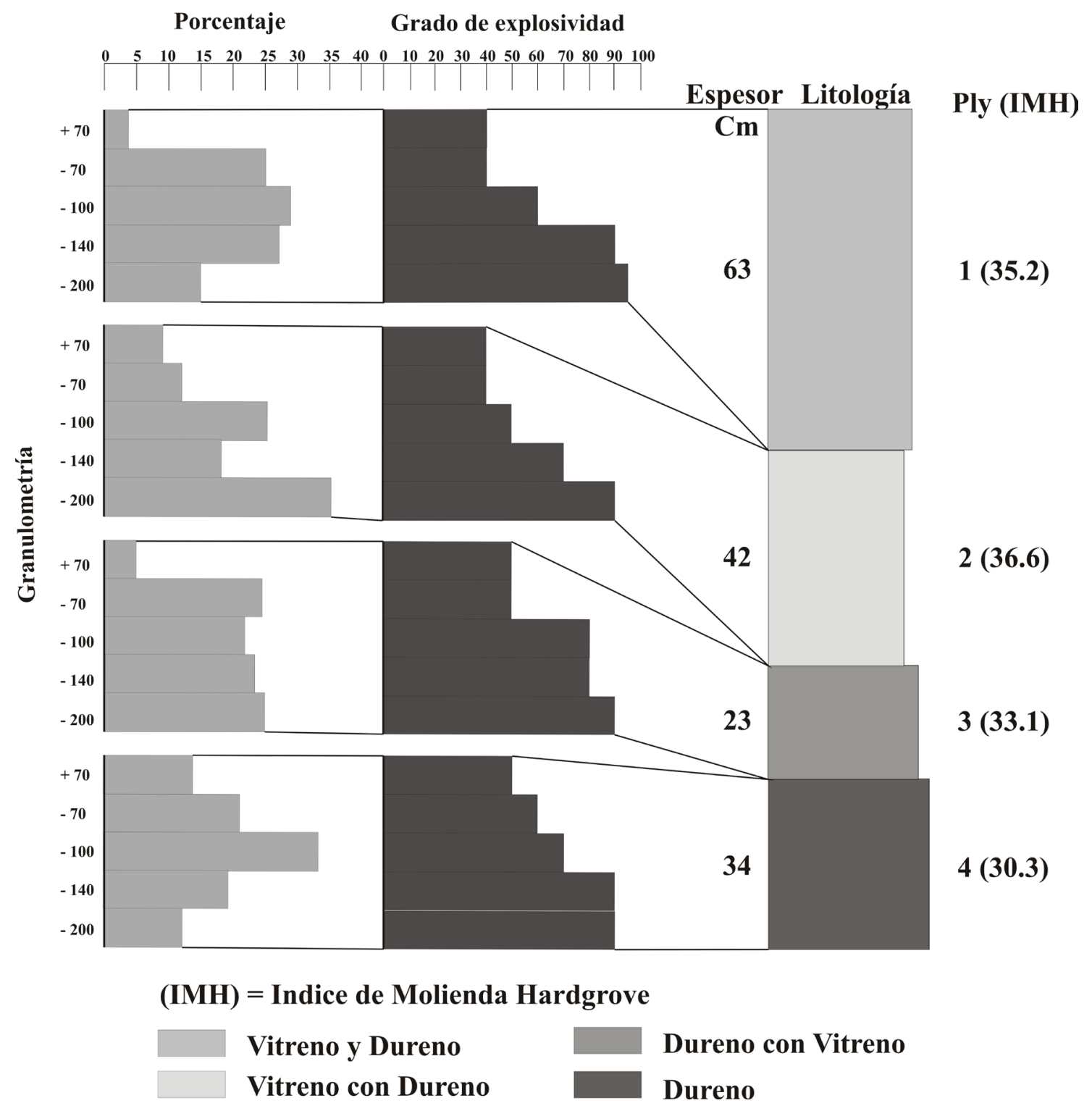

Figura 3. Relación entre la granulometría, el índice de molienda hardgrove y el índice de explosividad del polvo de carbón a lo largo de un manto en una Mina en Amagá

Si se tienen carbones con similar composición petrográfica, el índice de molienda es muy importante en la explosividad del carbón. Así las muestras que son más blandas tienen la posibilidad de generar mayor porcentaje de partículas finas y por lo tanto mayor grado de explosividad, y a su vez necesitarán mayor inertización, (Véase figura 4).

\subsection{Pruebas de ignición del carbón}

3.4.1 Relación entre la temperatura de ignición el tiempo de quema y la granulometría del polvo de carbón
Como se puede ver en la Figura 5, el manto se puede dividir en dos partes con características diferentes, la parte inferior que presenta temperaturas de ignición $\left(350-300{ }^{\circ} \mathrm{C}\right) \mathrm{y}$ tiempos de quema en la fracción pasante malla $200,(<200 \mathrm{~min})$, mientras que la parte superior del manto tiene temperaturas de ignición (400 $-300{ }^{\circ} \mathrm{C}$ ) y tiempos de quema en la fracción pasante malla $200,(>200 \mathrm{~min})$. Igualmente se observa que la granulometría está directamente relacionada a la temperatura de ignición y al tiempo de quemado, así, en general los tamaños menores a malla 200 son los que 
tienen temperatura de ignición y los tiempos de quemado más bajos. Esto es especialmente notable en las muestras de la parte inferior del manto (ply 3 y 4) que son más ricos en dureno.

\section{Relación del Indice de Molienda Hardgrove y el \% de inertización}

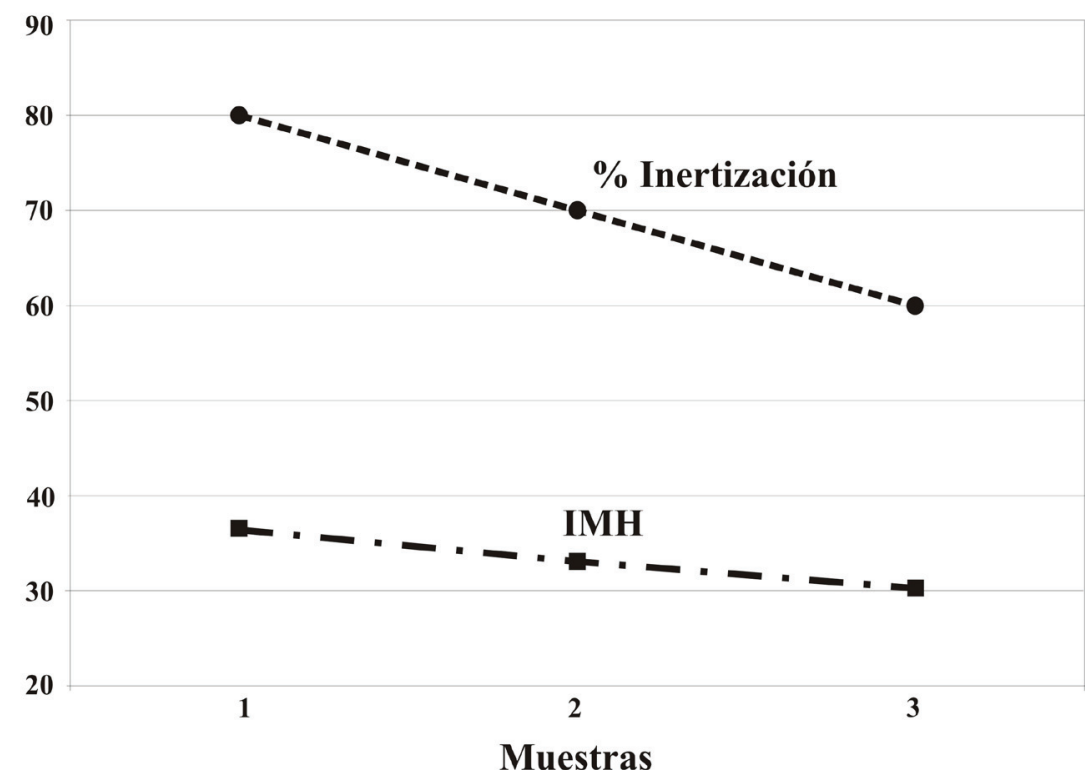

Figura 4. Relación del índice de molienda hardgrove con el porcentaje de inertización del polvo de carbón de un Manto de una Mina en Amagá.
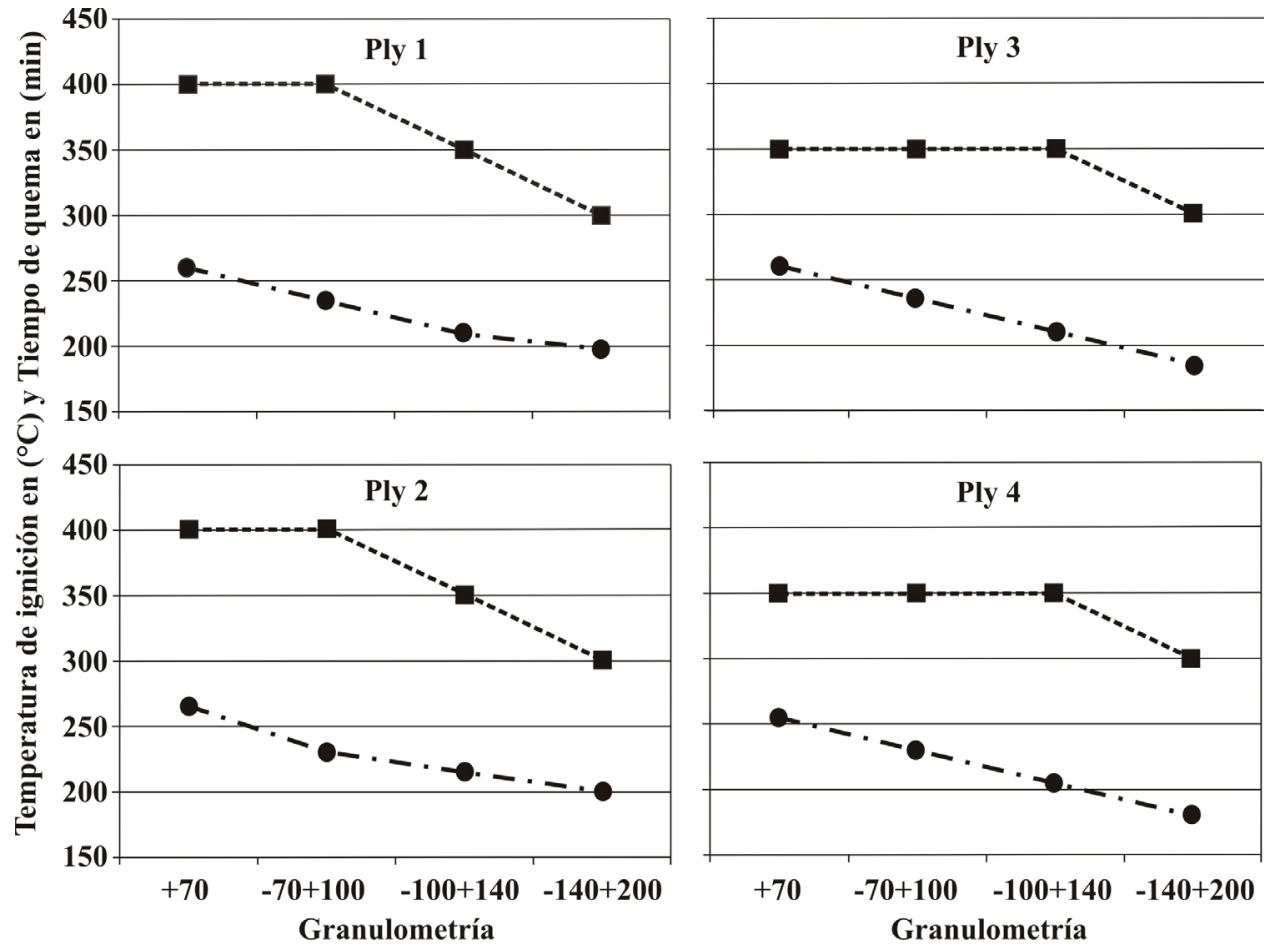

Figura 5. Relación entre la temperatura de ignición, el tiempo de quema, y la granulometría del polvo de carbón de los diferentes plies de un Manto de una Mina en Amagá. — Temperatura de ignición en (), • Tiempo de quema en (min) 


\subsection{Análisis petrográficos}

Los porcentajes de liptinitas son indicadores de autocombustión en los carbones de Amagá, dado que estos macerales, y en el caso especial de las muestras analizadas, presentan gotas de aceite que serían un aporte importante al proceso exotérmico generando fácil ignición del carbón y con mayor razón del polvo de carbón. (Véase figura 6).

En la Tabla 5, se puede evidenciar la comparación de los resultados obtenidos por medio de los análisis petrográficos con las pruebas de la temperatura de ignición, y el tiempo de quemado. Las muestras que presentan mayor susceptibilidad a la combustión espontánea del carbón son las que corresponden al ply 3 y 4 , ya que son las que tienen mayor contenido de liptinitas.
Tabla 5. Resultados de análisis petrográficos de los plies de un Manto de una Mina en Amagá

\begin{tabular}{ccccc}
\hline $\begin{array}{c}\text { Muestra } \\
\text { (manto) }\end{array}$ & $\begin{array}{c}\text { Vitrinita } \\
(\%)\end{array}$ & $\begin{array}{c}\text { Liptinita } \\
(\%)\end{array}$ & $\begin{array}{c}\text { Interinita } \\
(\%)\end{array}$ & $\begin{array}{c}\text { Minerales } \\
(\%)\end{array}$ \\
\hline M-1 Ply 1 & 67 & 24 & 8 & 1 \\
M-1 Ply 2 & 53 & 26 & 15 & 6 \\
M-1 Ply 3 & 60 & 34 & 5 & 1 \\
M-1 Ply 4 & 56 & 35 & 7 & 2 \\
\hline
\end{tabular}

\subsubsection{Relación de la composición maceral del carbón y el grado de explosividad}

Cuando se tienen carbones con diferente composición petrográfica, el índice de molienda pasa a un segundo plano, pues los componentes enriquecidos en hidrógeno generarían carbones con mayor susceptibilidad a la explosión. (Véase figuras 6 y 7 ).

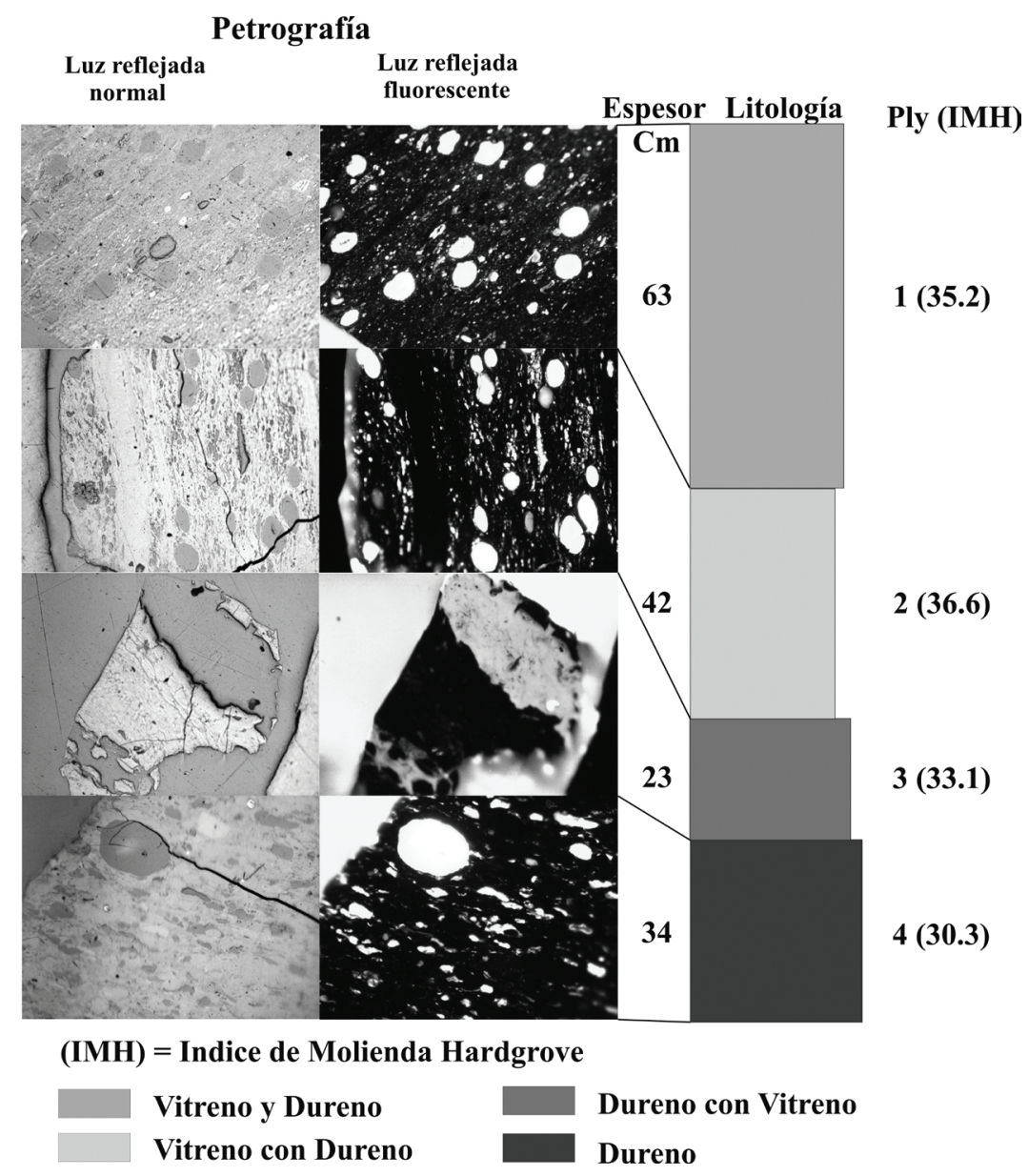

Figura 6. Relación de la composición maceral con el índice de molienda hardgrove a lo largo de un manto de una en Amagá. 

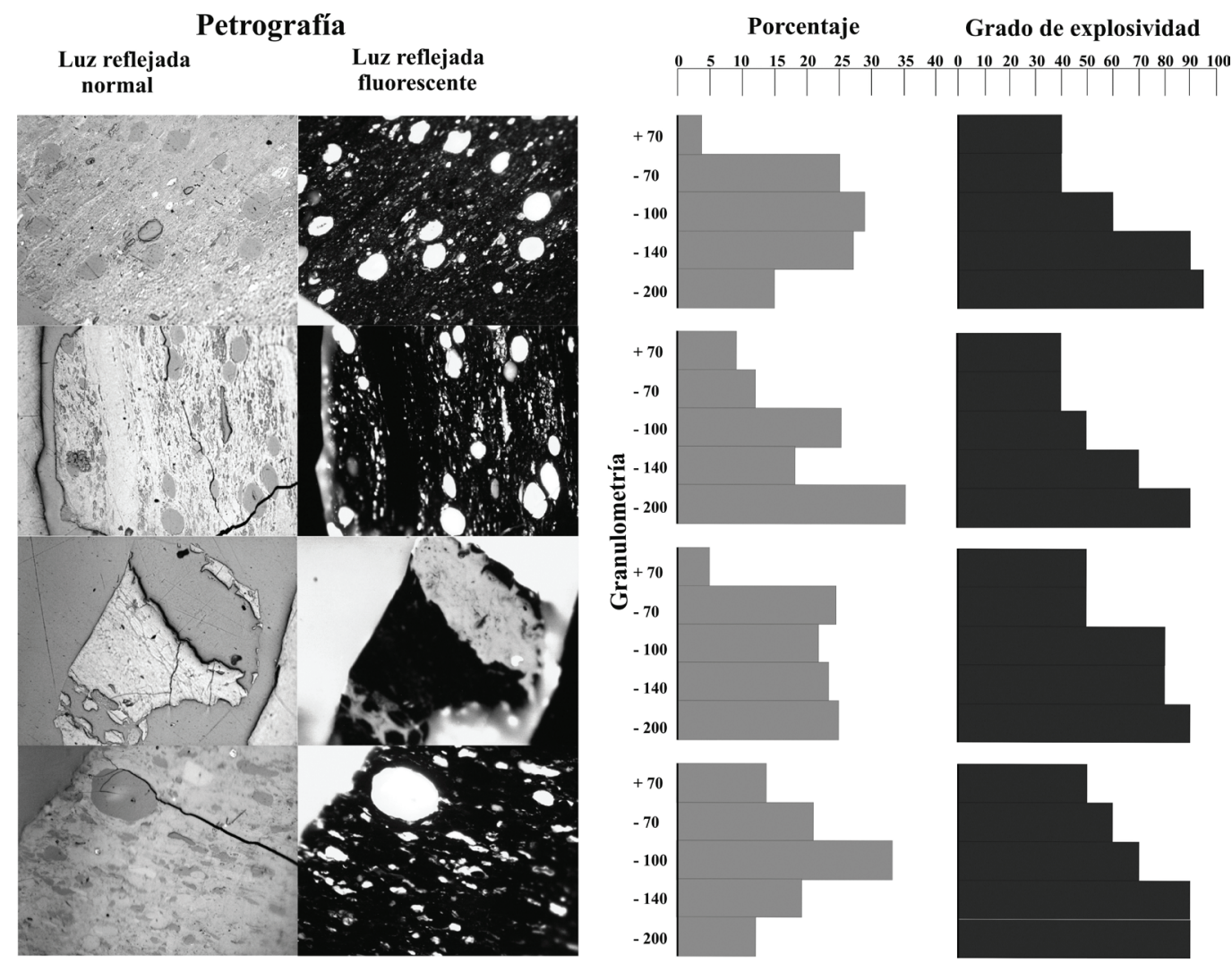

Figura 7. Relación entre la composición maceral, la distribución del tamaño de grano del polvo de carbón y el grado de explosividad de este.

\subsection{Análisis de explosividad de polvos de carbón}

Inicialmente, se realizaron los análisis generales de explosividad de las muestras tomadas en cada uno de los plies del un manto de una mina en Amagá. Posteriormente se tomaron en otro lugar de la mina las muestras de los plies de otro de los mantos Capotera y del que ya se había tomado antes. Los resultados obtenidos en esta prueba se muestran en la Tabla 6, donde los respectivos indicadores de explosividad son: Red $=$ Rojo, altamente explosivo; Yellow $=$ Amarillo, medianamente explosivo, Green $=$ Verde, no explosivo. A partir de estos datos es posible calcular la cantidad de material inertizante a aplicar en la mina para minimizar el riesgo de explosión por polvos de carbón, cabe anotar que para este caso se usó polvo de caliza.

De los resultados de la tabla 6 se puede observar la variación vertical y lateral de los mantos de carbón con respecto a la susceptibilidad a la explosión, especialmente en el caso de un Manto, pues en un sector de la mina, la inertización varía entre 75 y $85 \%$, y en otro sector de la misma mina la inertización varía entre 50 y $65 \%$. Para uno de los mantos, esta inertización varía entre 65 y $85 \%$. Lo anterior indica que no es suficiente con caracterizar en un solo punto el polvillo producido en la mina, porque es posible que se tome un sitio donde el manto no es tan explosivo, mientras en otro lugar el mismo manto puede ser explosivo. Además hay que tener en cuenta el tamaño de grano y la composición maceral predominante para que se inicie la autocombustión del polvo de carbón, lo que a su vez acelera la explosión.

Los resultados indican que todas las muestras de polvos finos de los plies de los carbones analizados son explosivas y que requieren de un alto porcentaje de caliza en polvo que varía entre 55 y $85 \%$ para su inertización, eso significa que se requiere entre 55 y $85 \mathrm{~g}$ de caliza en polvo y de 45 a $15 \mathrm{~g}$ de polvo de carbón para que la mezcla sea inerte y no haya riesgo de explosión por polvillo de carbón. 
Tabla 6. Resultados de explosividad de polvo de carbón de muestras de ply de los mantos Capotera y 1 de una Mina en Amagá

\begin{tabular}{|c|c|c|c|c|c|c|}
\hline \multirow{2}{*}{ Mina en Amagá } & \multicolumn{3}{|c|}{ INDICADOR DE EXPLOSIVIDAD } & \multirow{2}{*}{$\begin{array}{l}\% \text { de } \\
\text { inertización }\end{array}$} & \multirow{2}{*}{$\begin{array}{c}\% \text { P.C } \\
\% \text { de polvo de } \\
\text { carbón }\end{array}$} & \multirow{2}{*}{$\begin{array}{c}\% \text { P.R } \\
\% \text { de polvo de } \\
\text { roca }\end{array}$} \\
\hline & Red & Yellow & Green & & & \\
\hline *Manto 1 Ply 1 & 50 & $60-70$ & 75 & 75 & 25 & 75 \\
\hline *Manto 1 Ply 2 & 50 & $60-70$ & 80 & 80 & 20 & 80 \\
\hline *Manto 1 Ply 3 & 50 & $60-80$ & 85 & 85 & 15 & 85 \\
\hline *Manto 1 Ply 4 & 50 & $60-70$ & 75 & 75 & 25 & 75 \\
\hline Capotera ply 1 & 50 & $60-75$ & 80 & 80 & 20 & 80 \\
\hline Capotera ply 2 & 50 & $55-65$ & 70 & 70 & 30 & 70 \\
\hline Capotera ply 3 & 50 & $70-80$ & 85 & 85 & 15 & 85 \\
\hline Capotera ply 4 & 50 & $55-60$ & 65 & 65 & 35 & 65 \\
\hline Capotera ply 5 & 50 & $55-65$ & 70 & 70 & 30 & 70 \\
\hline Capotera ply 6 & 50 & $55-70$ & 80 & 80 & 20 & 80 \\
\hline Manto 1 ply 1 & 50 & 50 & 55 & 55 & 45 & 55 \\
\hline Manto 1 ply 2 & 50 & $55-65$ & 70 & 70 & 30 & 70 \\
\hline Manto 1 ply 3 & 50 & 50 & 55 & 55 & 45 & 55 \\
\hline Manto 1 ply 4 & 50 & $55-60$ & 65 & 65 & 35 & 65 \\
\hline Manto 1 ply 5 & 50 & 50 & 55 & 55 & 45 & 55 \\
\hline
\end{tabular}

*Muestras tomadas en otra fecha diferente en Amagá

En total se tomaron 40 muestras de polvos de carbón de minas subterráneas de Norte de Santander, Amagá, Boyacá y Cundinamarca.

Los resultados indican, que la mayoría de los carbones de Norte de Santander presentan explosividades muy altas varían entre $70 \mathrm{y}$ $95 \%$. En el caso de los carbones de Estados Unidos, los carbones más explosivos están como máximo en el rango 80 y $85 \%$ (Cashdollar, 2010) en ausencia de metano. De las 21 muestras analizadas en esta zona el $67 \%$ están por encima del $90 \%$ y el restante $33 \%$ está entre 65 y $70 \%$, esto significa que la gran mayoría de las muestras a pesar de ser carbones coquizables con contenidos de materia volátil menor que los carbones térmicos, tienen mayor contenido de hidrocarburos libres y producen tamaños de partículas más finas (polvos untuosos). Por lo tanto cuando se habla de explosividad de polvo de carbón no es suficiente con los análisis próximos, sino que es necesario mirar la composición petrográfica, para identificar la presencia o no de hidrocarburos libres, lo que está también relacionado con la susceptibilidad de éstos carbones a producir tamaños de partículas más finas.

Para el caso de los carbones de Amagá si es válido que a mayor contenido de materias volátiles es mayor la explosividad del polvo de carbón ya que los carbones afectados térmicamente por cuerpos intrusivos, presentan menor contenido de hidrocarburos libres y a diferencia de los de Norte de Santander, los tamaños de partículas son mucho mayores. De las muestras analizadas en esta cuenca e Amagá, el $21 \%$ presenta relativamente baja explosividad, menor al $50 \%$ de inertización, $(<50 \mathrm{~g}$ de polvo de caliza por $>50 \mathrm{~g}$ de polvo de carbón), mientras que el 79 $\%$ restante varía entre 70 y $90 \%$ de inertización, $(<70-90$ g de polvo de caliza por $<30-10$ g de polvo de carbón), y prácticamente ninguna está por encima de este valor.

\section{Conclusiones}

En la región de Amagá, las minas secas y pulverulentas, que explotan carbones térmicos con altos contenidos de materias volátiles, $(>$ $45 \%)$ requieren los más altos porcentajes de inertización (mayores a $80 \%$ ). Por el contrario, las minas que tienen mantos de carbón de mayor rango, con materias volátiles $(<40 \%)$ presentan bajos porcentajes de inertización (menor del 50 $\%)$.

En las muestras de carbón analizadas en Norte de Santander, los porcentajes de inertización variaron entre 65 y $95 \%$, pero predominan 
las muestras que requieren porcentajes de inertización de más del $80 \%$. Ello se debe al menor tamaño de grano del polvo de estas minas y a la presencia de hidrocarburos libres que hacen que éstas sean las más explosivas de todas las muestras analizadas.

Los resultados de los análisis indican que cuando los contenidos de partículas $>60 \%$ está por debajo de 75 micras, el peligro de explosión de dichos polvos es muy alto y sería difícil manejarlos únicamente adicionando agua, ya que se requieren porcentajes de inertización de más del $80 \%$.

Debido a la gran variación vertical y lateral de la materia orgánica y la materia mineral de los mantos de carbón, no es suficiente hacer las medidas de explosividad en un solo punto de la mina. Además es necesario tener en cuenta las variaciones en la composición maceral, ya que está directamente relacionada con el grado de explosividad del polvo de carbón, como es el caso de la abundancia de macerales del grupo de la liptinita.

Es necesario realizar pruebas de explosividad con los tamaños reales de grano del polvo de carbón en las minas. Cuando se tienen herramientas de corte directo del carbón como cepillos y rozadoras, como parte de la minería mecanizada, se tendrán partículas más finas, que cuando es arranque manual, o con perforación y voladuras.

Los análisis y resultados mostrados acá tienen que ver con las condiciones intrínsecas del carbón. Asuntos extrínsecos como concentraciones, fuentes de ignición, formación de nubes, tamaño de grano real dependiente del método de arranque, cantidad de oxígeno, entre otros, tienen que ver con la operación de la mina, tema que se recomienda sea también estudiado.

\section{Referencias bibliográficas}

ASTM 2009. Standard Specification for Wire Cloth and Sieves for Testing Purposes. West Conshohocken, Pennsylvania 19428-2959, United States: ASTM International.
Baquero M. K. (2010). Análisis de los factores que influyen en la combustión espontánea del carbón en la Mina San Fernando, Amagá. Universidad Nacional de Colombia, Sede Medellín, Facultad de Minas. 58 p.

Cashdollar, K. L., Sapko, M. J., Weiss E. S., Harris M. L., Man, C-K., Harteis S. P., \& Green G. M. (2010). Recommendations for a New Rock Dusting Standard to Prevent Coal Dust Explosions in Intake Airways. Report of Investigations 9679. Department of Health and Human Services Centers for Disease Control and Prevention National Institute for Occupational Safety and Health, 59 p.

Cashdollar K. L. (2000). Overview of dust explosibility characteristics. Journal of Loss Prevention in the Process Industries (13), 183199.

Castro W., \& Martínez C. (1999). Prevención de golpes de polvo en minas de carbón mediante la neutralización con material estéril, Revista DYNA, (126), 43-59.

Castro W., \& Martínez C. (1998). Riesgo potencial de explosiones de polvo combustible en minas subterráneas de carbón, Revista DYNA, (124), 37-53.

Castro W., \& Martínez C. (1997). Combustión espontánea en macizos de carbón, Revista DYNA, (123), 43-50.

CFR Code of Federal Regulations (2011), 30, parts 1 to 199. Office of Federal Register. Washington, D C.

Cybulski WG. (1975). Coal dust explosions and their suppression. Translated from Polish. Warsaw, Poland: National Center for Scientific, Technical and Economic Information. NTIS No. TT 73-54001.

Dobroski H Jr., Stephan CR, Conti RS. (1996). Historical summary of coal mine explosions in the United States. 1981-1994. Pittsburgh, PA: U.S. Department of the Interior, Bureau of Mines, IC 9440. 
Harris M., Sapko M., Cashdollar K., Verakis H. (2008). Field evaluation of the coal dust explosibility meter (CDEM), SME Annual meeting. p. 1-5.

Lebecki K. (1991). Gas dynamics of coal dust explosion - theory and experiment. In: Proceedings of the 24th International Conference of Safety in Mines Research Institutes (Donetsk, USSR) 1, 357-373.

Light, T.E., Herndon, R.C., Guley, A.R. Jr., Cook, G.L., Odum, M.A., Bates, R.M. Jr., Schroeder, M.E., Campbell, C.D., Pruitt, M.E. (2007). Report of investigation, fatal underground coal mine explosions, May 20, 2006. Darby mine No. 1, Kentucky Darby LLC, Holmes Mill, Harlan County, Kentucky, ID No. 15-18185. Arlington, VA: U.S. Department of Labor, Mine Safety and Health Administration.

McKinney, R., Crocco, W., Stricklin, K.G., Murray, K.A., Blankenship, S.T., Davidson, R.D., Urosek, J.E., Stephan, C.R., \& Beiter, D.A. (2002). Report of investigation, fatal underground coal mine explosions, September 23, 2001. No. 5 Mine, Jim Walters Resources, Inc., Brookwood, Tuscaloosa County, Alabama, ID No. 01-01322. Arlington, VA: U.S. Department of Labor, Mine Safety and Health Administration.
Michelis J. (1996). Large scale experiments with coal dust explosions in connection with road-Tjunctions. In: The Seventh International Colloquium on Dust Explosions; part of the International Symposium on Hazards, Prevention and Mitigation of Industrial Explosions (Bergen, Norway, June 23-26, 1996). Christian Michelsen Research, p. 8.50 8.59 .

Michelis, J., Margenburg, B., Müller, G., \& Kleine, W. (1987). Investigations into the buildup and development conditions of coal dust explosions in a 700-m underground gallery. In: Cashdollar KL, Hertzberg M, eds. Industrial dust explosions. West Conshohocken, PA: American Society for Testing and Materials (ASTM), Special Technical Publication (STP) 958, p. 124-137.

Reed, D., \& Michelis, J. (1989). Comparative investigation into explosibility of brown coal and bituminous coal dust in surface and underground test installations. In: Proceedings of the 23rd International Conference of Safety in Mines Research Institutes (Washington, DC, September 11-15, 1989). Pittsburgh, PA: U.S. Department of the Interior, Bureau of Mines, $p$. 941-964. 